\title{
The relationship between epicardial adipose tissue and arterial stiffness in patients with rheumatoid arthritis
}

\author{
Cristian Vasile Petra ${ }^{1}$, Adriana Albu², Cristina Pamfil ${ }^{1}$, Maria Magdalena Tămaș ${ }^{1}$, Ștefan \\ Cristian Vesa ${ }^{3}$, Simona Rednic ${ }^{1}$
}

${ }^{1}$ Rheumatology Department, ${ }^{2} 2^{\text {nd }}$ Internal Medicine Department, ${ }^{3}$ Pharmacology, Toxicology and Clinical Pharmacology Departament, "Iuliu Hațieganu" University of Medicine and Pharmacy, Cluj-Napoca, Romania

\begin{abstract}
Aim: To evaluate the relationship between epicardial adipose tissue (EAT) and arterial stiffness (AS) in patients with rheumatoid arthritis (RA), considering cardiovascular risk factors and disease characteristics. Material and methods: A total of 84 RA patients were included in this cross-sectional study. EAT and carotid intima-media thickness (cIMT) were measured ultrasonographically while aortic pulse wave velocity (aPWV), the main AS parameter, was determined using an oscillometric device. Results: Mean duration of RA was $12 \pm 9.5$ years and disease activity score was $4.3 \pm 1.4$, as assessed by Disease Activity Score-28 using C-reactive protein (DAS-28 CRP). The correlation analysis displayed a significant positive correlation between cIMT, aPWV and EAT ( $\mathrm{r}=0.037, \mathrm{p}<0.001 ; \mathrm{r}=0.338, \mathrm{p}=0.002$ and $\mathrm{r}=0.317, \mathrm{p}=0.003)$. When a cutoff value of aPWV $\geq 10 \mathrm{~m} / \mathrm{s}$ was established, patients with increased aPWV had significantly higher body mass index ( $\mathrm{p}=0.04)$, waist circumference $(\mathrm{p}=0.01)$, triglycerides $(\mathrm{p}=0.04)$, EAT $(\mathrm{p}<0.001)$, hypertension $(\mathrm{p}=0.03)$ and marginally C-reactive protein $(C R P)$ $(p=0.05)$. Multivariate regression analysis showed that hypertension $(p=0.033)$, increased CRP $(p=0.016)$ and EAT $(p=0.005)$ are the only independent predictors for increased aPWV. Conclusions: Our study found that increased AS independently correlated with EAT in patients with RA. Although the evaluation of these two parameters awaits further evidence to be included in the risk algorithms for CVD prevention, their role in patients with inflammatory diseases may be even more significant than in the general population.
\end{abstract}

Keywords: rheumatoid arthritis; subclinical atherosclerosis; carotid intima-media thickness; arterial stiffness; epicardial adipose tissue

\section{Introduction}

Rheumatoid arthritis (RA) is a very common autoimmune inflammatory disease [1] and is associated with an enhanced cardiovascular risk [2], besides joint involve-

Received 26.04.2019 Accepted 26.08.2019

Med Ultrason

2019, Vol. 21, No 4, 427-434

Corresponding author: Adriana Albu

$2^{\text {nd }}$ Internal Medicine Department, University of Medicine and Pharmacy "Iuliu Hațieganu"

2-4 Clinicilor Street,

400006, Cluj-Napoca, Romania

E-mail: albumed2@gmail.com ment. RA per se is now regarded as an independent risk factor for cardiovascular disease (CVD) [3].

The high frequency of traditional cardiovascular risk factors contributes only partly to the increased cardiovascular (CV) morbidity and mortality in RA, the high systemic inflammatory burden representing its key driver [4]. Due to the complexity of the proatherogenic processes involved, including chronic inflammation, genetic determinants, rheumatoid cachexia, the lipid paradox or the prothrombotic state, classical CVD risk calculators fail to accurately classify RA patients into risk groups and to predict future CV events [5]. Moreover, there is still a knowledge gap in how to quantify the exact impact 
of RA on the different stages of atherosclerosis, such as endothelial dysfunction, arterial wall hypertrophy and plaque formation [6].

It was demonstrated that excessive visceral adiposity participates in the pathogenesis of CVD [7]. RA patients are more prone to accumulate visceral adipose tissue than controls of similar body mass index (BMI) [8]. Epicardial adipose tissue (EAT) strongly correlates with general visceral adiposity [9] and has recently emerged as an index of cardiometabolic risk [10]. In non-RA patients, EAT has been independently associated with coronary artery disease [11], increased carotid intima-media thickness (cIMT) [12] and arterial stiffness (AS) [13]. Very few studies have evaluated EAT in RA. Although magnetic resonance imaging (MRI) represents the gold standard imaging technique to quantify total EAT, the echocardiographic measurement of EAT thickness is an easy-to-use, non-invasive and reliable evaluation, which correlates with the MRI findings [14].

The ultrasonographic measurements of cIMT and plaque assess subclinical atherosclerosis and represent the most widely used non-invasive imaging tool to determine CVD risk [15]. Increased values of cIMT and plaque presence predict future ischemic cardiac events and stroke in large population-based studies [16]. In RA, a recent meta-analysis reported thicker cIMT and faster plaque progression compared to healthy controls [17].

Increased AS represents an early marker of arteriosclerosis [18] and may serve as a screening method for the detection of pre-clinical cardiovascular disease [19]. Assessment of the mechanical properties of large arteries has become of great interest, as studies have shown that these parameters improve the prediction for future $\mathrm{CV}$ events in non-RA populations [20]. Chronic inflammation may play a key role in the early vascular aging in patients with inflammatory disorders [21]. Aortic pulse wave velocity (aPWV) is a simple, non-invasive measure of vascular compliance and a validated surrogate marker for AS. Along with cIMT and carotid plaque assessment, aPWV was predictive of CV events in a cohort of RA patients [22].

To the best of our knowledge, no study has to date addressed the relationship between EAT and AS in RA populations. Therefore, we designed the present study to evaluate and compare these two parameters, along with other CVD risk factors in patients with RA.

\section{Material and methods}

\section{Study population}

This cross-sectional study included 84 consecutive RA patients who fulfilled the 2010 American College of Rheumatology (ACR)/European League against Rheu- matism (EULAR) classification criteria [23]. The exclusion criteria were any history of atherosclerotic CV disease (including coronary heart disease, stroke and peripheral arterial disease), heart failure, atrial fibrillation, moderate to severe valvular disease, diabetes mellitus, acute or chronic renal failure, malignancy and any active infectious disease. The study protocol was approved by the local Ethics Committee and all subjects provided their informed consent.

\section{Clinical evaluation}

We recorded demographic characteristics such as age, disease duration, smoking status, associated diseases, current medication and performed a detailed clinical assessment. Metabolic syndrome was defined according to the International Diabetes Federation criteria [24]. Hypertension was diagnosed based on values equal to or greater than $140 \mathrm{mmHg}$ for the systolic pressure and/ or $90 \mathrm{mmHg}$ for the diastolic pressure or current antihypertensive therapy [25]. Disease activity was calculated based on Disease Activity Score-28 using C-reactive protein (DAS28-CRP) [26].

\section{Laboratory measurements}

Blood samples were withdrawn from the antecubital vein after a 12 hours fasting period. Biochemical parameters, including complete blood count, lipid profile, glucose, erythrocyte sedimentation rate (ESR) were measured by standard methods (Cobas Mira Plus analyzer, Hoffman La Roche, Switzerland) on the same day. CRP and IgM rheumatoid factor (RF, negative $<32 \mathrm{UI} / \mathrm{ml}$ ) were quantified by automated turbidimetry, whereas anti-cyclic citrullinated peptide antibodies (anti-CCP, negative $<5$ $\mathrm{UI} / \mathrm{ml}$ ) by enzyme-linked immunosorbent assay (ELISA).

Baseline echocardiography and epicardial adipose tissue measurements

The echocardiographic examinations were performed using a General Electric-Logiq S7 machine, with a 3.5 $\mathrm{MHz}$ transducer by an experienced cardiac investigator (more than 15 years of experience). All RA patients were examined in left lateral decubitus by two-dimensional echocardiography, M-mode and Doppler imaging. The sizes of cardiac cavities and wall thicknesses were determined individually. EAT was identified as the echo-free area between the outer wall of the myocardium and the visceral layer of the pericardium. EAT thickness was measured in parasternal long-axis view on the free wall of the right ventricle, perpendicularly to the aortic annulus. The measurements were performed at end-diastole in three cardiac cycles, using the average for statistical analysis.

\section{Carotid ultrasound measurements}

Carotid arteries were examined bilaterally with the same machine and by the same investigator as echocardiography, using high-resolution $12 \mathrm{MHz}$ linear-array 
transducer. cIMT was measured longitudinally using B-mode ultrasonography with the image focused on the posterior (far) wall. We reported the mean values of three measurements taken $10 \mathrm{~mm}$ proximally to the bifurcation on each side. Pathological cIMT corresponded to values from 0.9 to $1.5 \mathrm{~mm}$ and plaque presence was defined as any focal echogenic structure of at least $0.5 \mathrm{~mm}$ or $50 \%$ of the surrounding vessel wall or demonstrated a thickness $\geq 1.5 \mathrm{~mm}$ that protrudes into the lumen [27].

Reproducibility for EAT and cIMT was assessed in 12 subjects who were examined by the same observer twice, one week apart, with a coefficient of intra-observer variation of $6 \%$ and $5.7 \%$, respectively.

\section{Arterial stiffness assessment}

Parameters of AS were assessed non-invasively using a clinically validated oscillometric device (Arteriograph, TensioMed, Budapest, Hungary) $[28,29]$ by an investigator with 10 years of experience. All measurements were performed in a quiet, temperature-controlled environment. Patients refrained from food and drink for 12 hours prior to the study and were examined after resting supine for at least 15 minutes. Values of aPWV $(\mathrm{m} / \mathrm{s})$, central and brachial augmentation indexes (\%) and other hemodynamic parameters were determined automatically with the integrated software, including only the mean value of the best assessment (with a standard deviation of less than 1.0 for aPWV).

\section{Statistical Analysis}

Statistical analysis was performed using the MedCalc Statistical Software version 18.11.3 (MedCalc Software bvba, Ostend, Belgium; https://www.medcalc. org; 2019). Comparisons between groups were performed using the Mann-Whitney or the chi-square test, whenever appropriate. Correlations between qualitative variables were tested using Spearman's rho correlation coefficient. The area under the receiver operating characteristics (AUROC) curves was used for determining the cut-off values for several variables regarding the association with increased aPWV. For cut-offs we calculated the sensitivity (Se), specificity (Sp) and their confidence interval (CI95\%). Multivariate analysis was performed using logistic regression. Regression coefficients, odds ratio (OR) and CI95\% were reported. A p value $<0.05$ was considered statistically significant.

\section{Results}

Demographic and clinical findings of the study population are presented in Table I and echocardiographic and vascular measurements are displayed in Table II.

All three parameters (EAT, cIMT and aPWV) were significantly positively correlated with each other. Fur-
Table I. Baseline characteristics of the study group

\begin{tabular}{ll}
\hline Characteristics & Values \\
\hline Age, years & $55.6 \pm 12.3$ \\
Women, n (\%) & $71(84.5)$ \\
BMI, kg/m² & $27.3 \pm 5.4$ \\
WC, cm & $92.5 \pm 12.9$ \\
TC, mg/dL & $204.1 \pm 46.3$ \\
HDL-C, mg/dL & $56 \pm 13.5$ \\
TG, mg/dL & $113.9 \pm 48.4$ \\
LDL-C, mg/dL & $127.6 \pm 39$ \\
Hgb, g/dL & $12.9 \pm 1.3$ \\
WBC, 10 $/$ L & $7.0 \pm 2.0$ \\
MetS, n (\%) & $19(22.6)$ \\
Dyslipidemia, n (\%) & $42(50)$ \\
Hypertension, n (\%) & $31(36.9)$ \\
Current smoking, n (\%) & $13(15.5)$ \\
R A features & \\
Disease duration, years & $12 \pm 9.5$ \\
ESR, mm/h & $16($ range $10-39.7)$ \\
CRP, mg/L & $5($ range 2-11) \\
DAS28-CRP & $4.3 \pm 1.4$ \\
RF positivity, n (\%) & $61(72.6)$ \\
Anti-CCP positivity, n (\%) & $67(79.8)$ \\
Treatment & \\
Prednisone, n (\%) & $22(26.2)$ \\
Synthetic DMARD, n (\%) & $74(88.1)$ \\
Biologic DMARD, n (\%) & $29(34.5)$ \\
\hline
\end{tabular}

*Values are expressed as mean $\pm \mathrm{SD}$; BMI = body mass index; $\mathrm{WC}=$ waist circumference; $\mathrm{TC}=$ total cholesterol; HDL-C $=$ high density lipoprotein cholesterol; TG $=$ triglycerides; LDL-C $=$ low density lipoprotein cholesterol; $\mathrm{Hgb}=$ hemoglobin; $\mathrm{WBC}=$ white blood cell; MetS = metabolic syndrome; ESR = erythrocyte sedimentation rate; $\mathrm{CRP}=\mathrm{C}$-reactive protein; $\mathrm{DAS} 28-\mathrm{CRP}=$ disease activity score 28 joints count-CRP; RF = rheumatoid factor; Anti$\mathrm{CCP}=$ anti-cyclic citrullinated peptide; $\mathrm{DMARD}=$ disease-modifying anti-rheumatic drug.

thermore, statistically significant correlations were observed between these parameters and age, BMI, waist circumference (WC) and CRP. However, the correlation between aPWV and BMI was weak and did not reach statistical significance $(\mathrm{r}=0.210, \mathrm{p}=0.054)$. Additionally, aPWV was correlated with disease duration $(\mathrm{r}=0.210, \mathrm{p}=0.054)$. No significant correlations were found between EAT, cIMT, aPWV and markers of inflammation (ESR, CRP) or dyslipidemia (LDL-C) (Table III).

We explored the relationship between several variables and aPWV, using a cut-off value of $10 \mathrm{~m} / \mathrm{s}$ between normal and increased aPWV [25]. Patients with increased aPWV had significantly higher BMI, WC, TG, 
hypertension and EAT, and at the threshold of statistical significance CRP, as shown in Table IV.

We calculated cut-offs for several continuous variables for the association with increased aPWV. For CRP we found a cut-off value $>4 \mathrm{mg} / \mathrm{L}$ (AUC 0.648 (CI95\% 0.536-0.749) to have Se 83.33\% (CI95\% 58.6-96.4) and Sp 56.06\% (CI95\% 43.3-68.3). For EAT the calculated cut-off value was $>0.21 \mathrm{~mm}$ (AUC 0.729 (CI95\% 0.620-0.820); Se 88.89\% (CI95\% 65.3-98.6), Sp 56.06\% (CI95\% 43.3-68.3). We constructed several predictive models for increased aPWV using logistic regression and including the variables which achieved statistical significance in the univariate analysis. In the multivariate regression analysis, only hypertension, increased CRP and EAT were found to be independent predictors for increased aPWV (Table V).

\section{Discussions}

The main result of the study was the identification of EAT as an independent predictor of increased AS. We also showed that EAT, cIMT and aPWV were positively correlated with each other in patients with RA. To the best of our knowledge, no other study has assessed these parameters altogether in this group of patients.

EAT, an important component of the visceral adipose tissue, surrounds and shares the same microcirculation with the coronary arteries, acting as a metabolically active organ which expresses various pro-inflammatory cytokines, such as tumor necrosis factor (TNF)- $\alpha$, interleukin (IL)-6 and resistin, all with vasculopathic effects [30]. Patients with established RA present a peculiar pattern of fat distribution, with loss of muscle tissue and normal subcutaneous fat mass but significant visceral fat gain, resulting in little or no change in BMI, the socalled "cachectic obesity" [31]. Thus, in patients with

Table II. Echocardiographic and vascular measurements

\begin{tabular}{ll}
\hline Cardiac measurements & \\
\hline EF, \% & $62.9 \pm 6.1$ \\
IVS, mm & 10 (range 10-12) \\
PW, mm & 11 (range 10-12) \\
LVEDD, mm & $45.4 \pm 5.5$ \\
LVESD, mm & $29.6 \pm 3.8$ \\
EAT, mm & $0.23 \pm 0.06$ \\
AS measurements & \\
aPWV, m/s & $8.7 \pm 1.9$ \\
aAix, \% & $31.9 \pm 14.8$ \\
PP, mmHg & $58.1 \pm 12.2$ \\
SBP, mmHg & $135 \pm 19.1$ \\
DBP, mmHg & $76.9 \pm 11.2$ \\
MAP, mmHg & $96.3 \pm 13.1$ \\
Carotid measurements & \\
cIMT, mm & $0.7 \pm 0.2$ \\
Presence of carotid plaques, $\mathrm{n}(\%)$ & $22(26.2)$ \\
Calcified carotid plaques, $\mathrm{n}(\%)$ & $10(11.9)$ \\
\hline
\end{tabular}

*Values are expressed as mean $\pm \mathrm{SD} ; \mathrm{EF}=$ ejection fraction; IVS $=$ interventricular septum end-diastolic diameter; $\mathrm{PW}=$ left ventricle posterior wal end-diastolic diameter; LVEDD = left ventricle enddiastolic diameter; LVESD = left ventricle end-systolic diameter; EAT $=$ epicardial adipose tissue; $\mathrm{AS}=$ arterial stiffness; $\mathrm{aPWV}=$ aortic pulse wave velocity; aAix = aortic augmentation index; $\mathrm{PP}=$ brachial pulse pressure; $\mathrm{SBP}=$ systolic blood pressure; $\mathrm{DBP}=$ diastolic blood pressure; MAP $=$ mean blood pressure; cIMT $=$ carotid intima-media thickness.

Table III. Correlation analysis between epicardial adipose tissue, aortic pulse wave velocity, carotid intima-media thickness and other variables

\begin{tabular}{lllllll}
\hline & \multicolumn{2}{c}{ EAT } & \multicolumn{2}{c}{ aPWV } & \multicolumn{2}{c}{ cIMT } \\
\cline { 2 - 7 } & $\mathbf{r}$ & $\mathbf{p}$ value & $\mathbf{r}$ & $\mathbf{p ~ v a l u e}$ & $\mathbf{r}$ & p value \\
\hline Age & 0.278 & $\mathbf{0 . 0 1}$ & 0.496 & $<\mathbf{0 . 0 0 1}$ & 0.568 & $<\mathbf{0 . 0 0 1}$ \\
BMI & 0.324 & $\mathbf{0 . 0 0 3}$ & 0.210 & 0.054 & 0.347 & $<\mathbf{0 . 0 0 1}$ \\
WC & 0.315 & $\mathbf{0 . 0 0 3}$ & 0.295 & $\mathbf{0 . 0 0 6}$ & 0.453 & $<\mathbf{0 . 0 0 1}$ \\
LDL-C & 0.050 & 0.648 & -0.028 & 0.800 & 0.027 & 0.809 \\
ESR & 0.080 & 0.469 & 0.116 & 0.289 & 0.015 & 0.891 \\
CRP & 0.113 & 0.314 & 0.090 & 0.421 & 0.170 & 0.127 \\
DAS28-CRP & -0.121 & 0.272 & 0.094 & 0.394 & -0.019 & 0.860 \\
Disease duration & 0.091 & 0.406 & 0.236 & $\mathbf{0 . 0 3 0}$ & 0.211 & 0.053 \\
EAT & & & 0.317 & $\mathbf{0 . 0 0 3}$ & 0.338 & $\mathbf{0 . 0 0 2}$ \\
aPWV & & & & & 0.374 & $<\mathbf{0 . 0 0 1}$ \\
\hline
\end{tabular}

$\mathrm{BMI}=$ body mass index; $\mathrm{WC}=$ waist circumference; $\mathrm{LDL}-\mathrm{C}=$ low density lipoprotein cholesterol; ESR = erythrocyte sedimentation rate; $\mathrm{CRP}=\mathrm{C}$-reactive protein; DAS28-CRP $=$ disease activity score 28 joints count- $\mathrm{CRP}$; EAT $=$ epicardial adipose tissue; aPWV $=$ aortic pulse wave velocity; aAix = aortic augmentation index; cIMT = carotid intima-media thickness. 
Med Ultrason 2019; 21(4): 427-434

Table IV. Comparison between clinical and paraclinical parameters in patients with normal and increased aortic pulse wave velocity

\begin{tabular}{llll}
\hline Variables & aPWV $<\mathbf{1 0 ~} \mathbf{~} / \mathbf{s}$ & aPWV $\geq \mathbf{1 0 ~} \mathbf{~} / \mathbf{s}$ & p value \\
\hline Age & $54.5(41.2-62.7)$ & $63(54-66)$ & 0.13 \\
BMI & $25.5(23.2-29)$ & $28.9(23.9-32.5)$ & $\mathbf{0 . 0 4}$ \\
WC & $91(81.2-98.5)$ & $95(89-111)$ & $\mathbf{0 . 0 1}$ \\
TG & $103(68.5-131.5)$ & $143(87-178)$ & $\mathbf{0 . 0 4}$ \\
TC & $189.5(169.5-222.5)$ & $207(171-250)$ & 0.13 \\
HDL-C & $53.5(46.2-59)$ & $56(39-63)$ & 0.53 \\
LDL-C & $115.5(100.5-135.5)$ & $141(104-147)$ & 0.14 \\
MetS & $13(19.7 \%)$ & $6(33.3 \%)$ & 0.22 \\
Hypertension & $20(30.3 \%)$ & $11(61.1 \%)$ & $\mathbf{0 . 0 3}$ \\
ESR & $16(10-37)$ & $11.7(6.5-18.5)$ & 0.45 \\
CRP & $0.4(0.1-1.2)$ & $9(5-11)$ & 0.05 \\
DAS-28 CRP & $4(3-5)$ & $5(4-5)$ & 0.27 \\
Disease duration & $9.5(5-15)$ & $12.5(8.2-22.7)$ & 0.17 \\
cIMT & $0.65(0.6-8)$ & $0.72(0.66-0.79)$ & 0.20 \\
Plaque presence & $14(21.2 \%)$ & $7(38.9 \%)$ & 0.14 \\
EAT & $0.21(0.19-0.27)$ & $0.26(0.23-0.31)$ & $<\mathbf{0 . 0 0 1}$ \\
\hline BI
\end{tabular}

$\mathrm{BMI}=$ body mass index $\mathrm{WC}=$ waist circumference; $\mathrm{TG}=$ triglycerides; $\mathrm{TC}=$ total cholesterol; HDL-C $=$ high density lipoprotein cholesterol; LDL-C = low density lipoprotein cholesterol; MetS = metabolic syndrome; ESR = erythrocyte sedimentation rate; $\mathrm{CRP}=\mathrm{C}$-reactive protein; DAS28-CRP = disease activity score 28 joints count- CRP; aPWV = aortic pulse wave velocity; cIMT = carotid intima-media thickness; EAT = epicardial adipose tissue. The Mann-Whitney test was used for quantitative variables and the chi-square test was used for qualitative variables.

Table V. Multivariate logistic regression analysis of parameters associated with increased aortic pulse wave velocity

\begin{tabular}{lllll}
\hline & Beta Coefficient & p value & OR & 95\% CI \\
\hline Hypertension & 1.432 & $\mathbf{0 . 0 3 3}$ & 4.188 & $1.12-15.61$ \\
Triglycerides & 0.641 & 0.396 & 1.898 & $0.43-8.34$ \\
CRP $>4 \mathrm{mg} / \mathrm{L}$ & 1.869 & $\mathbf{0 . 0 1 6}$ & 6.481 & $1.42-29.52$ \\
EAT $>0.21 \mathrm{~mm}$ & 2.390 & $\mathbf{0 . 0 0 5}$ & 10.909 & $2.05-57.8$ \\
\hline
\end{tabular}

$\mathrm{OR}=$ odds ratio $\mathrm{CI}=$ confidence interval $\mathrm{CRP}=\mathrm{C}$-reactive protein EAT $=$ epicardial adipose tissue.

chronic inflammatory diseases, EAT measurement could provide the most accurate and conclusive data regarding the degree of obesity and subsequent CV risk. However, very few studies have evaluated EAT in RA patients. Transthoracic echocardiographic assessment of EAT represents a simple, non-invasive, reproducible and readily available imaging technique. In a study by Iacobellis et al, echocardiographic EAT thickness showed good ultrasonographic concordance with MRI measurements and was the strongest independent variable correlated with abdominal visceral adipose tissue [14].

In agreement with other reports from the general population, we found that in RA patients EAT strongly correlates with age and other markers of obesity, such as BMI and WC [32,33]. Ormseth et al confirmed these anthropomorphic findings in a study performed on 162 RA patients, where EAT was measured by noncontrast cardiac computed tomography (CT) [34]. Similarly, they found no significant associations between EAT and inflammatory markers or DAS-28 CRP score. However, EAT thickness in RA patients was reported in two other studies to be independently associated with the DAS-28 CRP score [35], CRP and ESR, respectively [36]. Larger prospective studies are required to clarify the exact link between EAT and inflammation.

$\mathrm{AS}$ is one of the earliest detectable alterations of the vascular walls and an independent predictor of future CV events and all-cause mortality [37]. A meta-analysis based on 25 studies showed that a more severe inflammatory status impacts on AS measured by aPWV, augmentation index (Aix), and augmentation index normalized for a heart rate of 75 beats per minute (AIx@75) and that RA patients associated an increased AS compared with controls [38].

In bivariate analysis we found that aPWV correlated with age, disease duration, WC and BMI. In line with 
these findings, a recent study reported that each year of disease contributes to a higher rate of vascular aging than a year of life without RA [39]. On the contrary, Beyazal et al found no impact of disease duration on vascular function [40]. Caraba et al reported significant associations between low serum levels of vitamin $\mathrm{D}$ and disease activity, increased insulin resistance, and endothelial dysfunction (assessed by flow-mediated vasodilation, on brachial artery, using B-mode ultrasonography) in early RA patients with moderate and high disease activity [41].

The relation between EAT and AS has been addressed in studies performed both in the general population, as well as in subgroups of patients with hypertension, cardiovascular disease, post-menopausal women or nonalcoholic fatty liver disease [13,42-44], all reporting significant correlation. Choi et al evaluated EAT volume and coronary artery calcium (CAC) score and their relationship with AS by cardiac CT [45]. Interestingly, they reported EAT to be an independent predictor of increased PWV even in patients without CAC, suggesting an early systemic interplay between visceral adipose tissue and vascular stiffness, prior to any atherosclerotic changes in coronary vessels.

In our study, the association between AS and EAT was stronger than with other markers of obesity such as WC and BMI. EAT also showed a significant positive correlation with cIMT. Each of the two easily-applicable parameters, EAT ultrasound measurement and aPWV, may be taken into consideration and included into a novel CVD risk assessment tool (along with cIMT), after validation in broad study populations. Future research may evaluate a possible cause-effect relationship between these two parameters and identify inflammatory mediators involved in both EAT and AS.

A study performed on 107 untreated hypertensive patients reported that EAT was an independent factor for adverse changes in cIMT, flow-mediated dilatation (FMD) of the brachial artery and PWV [46]. Temiz et al provided further evidence that EAT correlated with cIMT and FMD in RA patients and suggested that EAT could serve as a surrogate marker for estimating the extent of vascular involvement [36]. However, AS may represent a more accurate marker of disease burden over time than FMD, as recent evidence indicated [47].

In rheumatic diseases, cIMT and plaque assessment by ultrasonography are the most widely used imaging markers of subclinical atherosclerosis and subsequent CVD risk. Screening for asymptomatic atherosclerotic plaques represent the only imagistic technique included in the EULAR recommendations for CVD risk evaluation in RA patients [48]. This simple, readily available ultrasound method was shown to reclassify RA patients into a more appropriate CVD risk group [49].

Although mean cIMT thickness did not exceed the pathological value of $0.9 \mathrm{~mm}$, plaque presence was relatively high in our study population (25.9\%). cIMT showed significant positive correlations with age and obesity markers, such as EAT (strongest correlation), BMI and WC. No correlation could be established with CRP, ESR, DAS-28 CRP, nor with the lipid profile, similar to other studies [40].

The present study has several limitations. Due to its cross-sectional design without a control group for comparison, no cause-effect relations can be drawn from our study. The fact that cardiac and carotid measurements were performed by a single investigator may also be considered a limitation. However, the intra-observer coefficients of variation in our study suggest good quality of measured data. Moreover, we determined EAT only by means of echography and did not compare it with other imaging techniques, such as CT or MRI. However, it has been shown that echocardiographic determination of EAT, which is an easy and non-invasive technique, is comparable to MRI measurements of EAT [9]. Another limitation was not assessing proinflammatory cytokines, supposed to be involved in the arterial walls alterations induced by increased EAT as described in the literature [30].

\section{Conclusions}

The present study demonstrates that increased AS is independently associated with EAT in RA patients. EAT a marker of visceral adiposity may be an important determinant of arteriosclerosis in RA and may thus contribute to the increased cardiovascular risk associated with this inflammatory disease.

\section{References}

1. Gibofsky A. Overview of epidemiology, pathophysiology, and diagnosis of rheumatoid arthritis. Am J Manag Care 2012;18(13 Suppl):S295-S302.

2. Aviña-Zubieta JA, Choi HK, Sadatsafavi M, Etminan M, Esdaile JM, Lacaille D. Risk of cardiovascular mortality in patients with rheumatoid arthritis: a meta-analysis of observational studies. Arthritis Rheum 2008;59:1690-1697.

3. Piepoli MF, Hoes AW, Agewall S, et al. 2016 European Guidelines on cardiovascular disease prevention in clinical practice: The Sixth Joint Task Force of the European Society of Cardiology and Other Societies on Cardiovascular Disease Prevention in Clinical Practice (constituted by representatives of 10 societies and by invited experts) Developed with the special contribution of the European Association for Cardiovascular Prevention \& Rehabilitation (EACPR). Eur Heart J 2016;37:2315-2381. 
4. Choy E, Ganeshalingam K, Semb AG, Szekanecz Z, Nurmohamed M. Cardiovascular risk in rheumatoid arthritis: recent advances in the understanding of the pivotal role of inflammation, risk predictors and the impact of treatment. Rheumatology (Oxford) 2014;53:2143-2154.

5. Arts EE, Popa C, Den Broeder AA, et al. Performance of four current risk algorithms in predicting cardiovascular events in patients with early rheumatoid arthritis. Ann Rheum Dis 2015;74:668-674.

6. Martín-Martínez MA, González-Juanatey C, Castañeda $\mathrm{S}$, et al. Recommendations for the management of cardiovascular risk in patients with rheumatoid arthritis: scientific evidence and expert opinion. Semin Arthritis Rheum 2014;44:1-8.

7. González N, Moreno-Villegas Z, González-Bris A, Egido J, Lorenzo Ó. Regulation of visceral and epicardial adipose tissue for preventing cardiovascular injuries associated to obesity and diabetes. Cardiovasc Diabetol 2017;16:44.

8. Santos MJ, Vinagre F, Canas da Silva J, Gil V, Fonseca JE. Body composition phenotypes in systemic lupus erythematosus and rheumatoid arthritis: a comparative study of Caucasian female patients. Clin Exp Rheumatol 2011;29:470 476.

9. Iacobellis G, Assael F, Ribaudo MC, et al. Epicardial fat from echocardiography: a new method for visceral adipose tissue prediction. Obes Res 2003;11:304-310.

10. Iacobellis G. Local and systemic effects of the multifaceted epicardial adipose tissue depot. Nat Rev Endocrinol 2015;11:363-371.

11. Erkan AF, Tanindi A, Kocaman SA, Ugurlu M, Tore HF. Epicardial Adipose Tissue Thickness is an Independent predictor of critical and Complex Coronary Artery Disease by Gensini and Syntax Scores. Tex Hear Inst J 2016;43:29-37.

12. Altin C, Sade LE, Gezmis E, et al. Assessment of Subclinical Atherosclerosis by Carotid Intima-Media Thickness and Epicardial Adipose Tissue Thickness in Prediabetes. Angiology 2016;67:961-969.

13. Kim BJ, Kim BS, Kang JH. Echocardiographic epicardial fat thickness is associated with arterial stiffness. Int J Cardiol 2013;167:2234-2238.

14. Iacobellis G, Ribaudo MC, Assael F, et al. Echocardiographic epicardial adipose tissue is related to anthropometric and clinical parameters of metabolic syndrome: a new indicator of cardiovascular risk. J Clin Endocrinol Metab. 2003;88:5163-5168.

15. Nezu T, Hosomi N, Aoki S, Matsumoto M. Carotid IntimaMedia Thickness for Atherosclerosis. J Atheroscler Thromb 2016;23:18-31.

16. Polak JF, Szklo M, Kronmal RA, et al. The value of carotid artery plaque and intima-media thickness for incident cardiovascular disease: the multi-ethnic study of atherosclerosis. J Am Heart Assoc 2013;2:e000087.

17. Wang P, Guan SY, Xu SZ, et al. Increased carotid intimamedia thickness in rheumatoid arthritis: an update metaanalysis. Clin Rheumatol 2016;35:315-323.

18. Cecelja M, Chowienczyk P. Arterial stiffening: Causes and consequences. Artery Res 2013;7:22-27.
19. Mozos I, Borzak G, Caraba A, Mihaescu R. Arterial stiffness in hematologic malignancies. Onco Targets Ther 2017;10:1381-1388.

20. Ben-Shlomo Y, Spears M, Boustred C, et al. Aortic pulse wave velocity improves cardiovascular event prediction: an individual participant meta-analysis of prospective observational data from 17,635 subjects. J Am Coll Cardiol 2014;63:636-646.

21. Dregan A. Arterial stiffness association with chronic inflammatory disorders in the UK Biobank study. Heart 2018;104:1257-1262.

22. Ikdahl E, Rollefstad S, Wibetoe G, et al. Predictive Value of Arterial Stiffness and Subclinical Carotid Atherosclerosis for Cardiovascular Disease in Patients with Rheumatoid Arthritis. J Rheumatol 2016;43:1622-1630.

23. Aletaha D, Neogi T, Silman AJ, et al. 2010 Rheumatoid arthritis classification criteria: An American College of Rheumatology/European League Against Rheumatism collaborative initiative. Arthritis Rheum 2010;62:2569-2581.

24. Alberti KG, Zimmet P, Shaw J. Metabolic syndrome - a new world-wide definition. A Consensus Statement from the International Diabetes Federation. Diabet Med 2006;23:469-480.

25. Williams B, Mancia G, Spiering W, et al. 2018 ESC/ESH Guidelines for the management of arterial hypertension. Eur Heart J 2018;39:3021-3104.

26. Prevoo ML, van 't Hof MA, Kuper HH, van Leeuwen MA, van de Putte LB, van Riel PL. Modified disease activity scores that include twenty-eight-joint counts. development and validation in a prospective longitudinal study of patients with rheumatoid arthritis. Arthritis Rheum 1995;38: 44-48.

27. Touboul PJ, Hennerici MG, Meairs S, et al. Mannheim carotid intima-media thickness and plaque consensus (20042006-2011). An update on behalf of the advisory board of the $3 \mathrm{rd}, 4$ th and 5 th watching the risk symposia, at the $13 \mathrm{th}$, 15th and 20th European Stroke Conferences, Mannheim, Germany, 2004, Brussels, Belgium, 2006, and Hamburg, Germany, 2011. Cerebrovasc Dis 2012;34:290-296.

28. Baulmann J, Schillings U, Rickert S, et al. A new oscillometric method for assessment of arterial stiffness: comparison with tonometric and piezo-electronic methods. J Hypertens 2008;26:523-528.

29. Echeverri D, Pizano A, Cabrales J, Moreno K. Validation of Central and Peripheral Non-Invasive Hemodynamic Variables Using an Oscillometric Method. High Blood Press Cardiovasc Prev 2017;25:65-77

30. Fitzgibbons TP, Czech MP. Epicardial and perivascular adipose tissues and their influence on cardiovascular disease: Basic mechanisms and clinical associations. J Am Heart Assoc 2014;3: e000582.

31. Kerekes G, Nurmohamed MT, González-Gay MA, et al. Rheumatoid arthritis and metabolic syndrome. Nat Rev Rheumatol 2014;10:691-696.

32. Shi KL, Qi L, Mao DB, et al. Impact of age on epicardial and pericoronary adipose tissue volume. Eur Rev Med Pharmacol Sci 2015;19:3257-3265. 
33. Rabkin SW. The relationship between epicardial fat and indices of obesity and the metabolic syndrome: a systematic review and meta-analysis. Metab Syndr Relat Disord 2014; $12: 31-42$.

34. Ormseth MJ, Lipson A, Alexopoulos N, et al. Association of epicardial adipose tissue with cardiometabolic risk and metabolic syndrome in patients with rheumatoid arthritis. Arthritis Care Res (Hoboken) 2013;65:1410-1415.

35. Alpaydin S, Buyukterzi Z, Akkurt HE, Y1lmaz H. Impaired left ventricular diastolic functions and thickened epicardial adipose tissue in rheumatoid arthritis patients is correlated with DAS-28 Score. Acta Cardiol Sin 2017;33:182-187.

36. Temiz A, Gökmen F, Gazi E, et al. Epicardial adipose tissue thickness, flow-mediated dilatation of the brachial artery, and carotid intima-media thickness. Herz 2015;40 Suppl 3:217-224.

37. Vlachopoulos C, Aznaouridis K, Terentes-Printzios D, Ioakeimidis N, Stefanadis C. Prediction of cardiovascular events and all-cause mortality with brachial-ankle elasticity index: a systematic review and meta-analysis. Hypertension 2012;60:556-562.

38. Ambrosino P, Tasso M, Lupoli R, et al. Non-invasive assessment of arterial stiffness in patients with rheumatoid arthritis: A systematic review and meta-analysis of literature studies. Ann Med 2015;47:457-467.

39. Vázquez-Del Mercado M, Gomez-Bañuelos E, ChavarriaAvila E, et al. Disease duration of rheumatoid arthritis is a predictor of vascular stiffness: a cross-sectional study in patients without known cardiovascular comorbidities: A STROBE-compliant article. Medicine (Baltimore) 2017;96:e7862.

40. Beyazal MS, Erdoğan T, Devrimsel G, et al. Relationship of osteoprotegerin to pulse wave velocity and carotid intimamedia thickness in rheumatoid arthritis patients. Z Rheumatol 2016;75:723-728.

41. Caraba A, Crişan V, Romoşan I, Mozoş I, Murariu M. Vitamin D Status, Disease Activity, and Endothelial Dysfunc- tion in Early Rheumatoid Arthritis Patients. Dis Markers. 2017;2017:5241012.

42. Al-Talabany S, Mordi I, Graeme Houston J, et al. Epicardial adipose tissue is related to arterial stiffness and inflammation in patients with cardiovascular disease and type 2 diabetes. BMC Cardiovasc Disord 2018;18:31.

43. Cabrera-Rego JO, Navarro-Despaigne D, Staroushik-Morel L, Díaz-Reyes K, Lima-Martínez MM, Iacobellis G. Association between endothelial dysfunction, epicardial fat and subclinical atherosclerosis during menopause. Clin Investig Arterioscler 2018;30:21-27.

44. Sunbul M, Agirbasli M, Durmus E, et al. Arterial stiffness in patients with non-alcoholic fatty liver disease is related to fibrosis stage and epicardial adipose tissue thickness. Atherosclerosis 2014;237:490-493.

45. Choi TY, Ahmadi N, Sourayanezhad S, Zeb I, Budoff MJ. Relation of vascular stiffness with epicardial and pericardial adipose tissues, and coronary atherosclerosis. Atherosclerosis 2013;229:118-123.

46. Kocaman SA, Durakoğlugil ME, Çetin M, Erdoğan T, Ergül E, Çanga A. The independent relationship of epicardial adipose tissue with carotid intima-media thickness and endothelial functions. Blood Press Monit 2013;18:8593.

47. Scanlon EM, Mankad R, Crowson CS, et al. Cardiovascular risk assessment in patients with rheumatoid arthritis: a correlative study of noninvasive arterial health testing. Clin Rheumatol 2017;36:763-771.

48. Agca R, Heslinga SC, Rollefstad S, et al. EULAR recommendations for cardiovascular disease risk management in patients with rheumatoid arthritis and other forms of inflammatory joint disorders: 2015/2016 update. Ann Rheum Dis 2016;76:17-28.

49. Semb AG, Ikdahl E, Hisdal J, Olsen IC, Rollefstad S. Exploring cardiovascular disease risk evaluation in patients with inflammatory joint diseases. Int $\mathrm{J}$ Cardiol 2016;223:331-336. 